\title{
Design and Evaluation of a Full-Wave Surface and Bottom-Detection Algorithm for LiDAR Bathymetry of Very Shallow Waters
}

Type: Peer reviewed journal article.

Status: Accepted February 8, 2019, published April 1, 2019 10.1016/j.isprsjprs.2020.03.015

Version: Manuscript accepted for publication.

Copyright: This work is licensed under a Creative Commons "Attribution-NonCommercialNoDerivatives 4.0 International" licence.

Bibliography: Roland Schwarz et al. "Design and evaluation of a full-wave surface and bottom-detection algorithm for LiDAR bathymetry of very shallow waters." In: ISPRS Journal of Photogrammetry and Remote Sensing 150 (Apr. 2019), pp. 1-10. DOI: 10.1016/ j.isprsjprs.2019.02.002 



\title{
Design and Evaluation of a Full-Wave Surface and Bottom-Detection Algorithm for LiDAR Bathymetry of Very Shallow Waters
}

\author{
R. Schwarz ${ }^{\mathrm{a}, \mathrm{b}, *}$, G. Mandlburger ${ }^{\mathrm{b}, \mathrm{c}}$, M. Pfennigbauer ${ }^{\mathrm{a}}$, N. Pfeifer ${ }^{\mathrm{b}}$ \\ ${ }^{a}$ Riegl Research Forschungs Gesellschaft mbH, 3580 Horn, Austria \\ ${ }^{b}$ Department of Geodesy and Geoinformation, Technische Universität Wien, Gußhausstr. 27-29, 1040 Vienna, Austria \\ ${ }^{c}$ Institute for Photogrammetry, University of Stuttgart, Geschwister-Scholl Str. 24D, 70174, Stuttgart Germany
}

\begin{abstract}
Airborne Laser Bathymetry (ALB) is an attractive technology for the measurement of shallow water bodies because of the high acquisition rate and high point densities that can be achieved. Of special interest is the application of ALB in non-navigable areas where the only alternatives are conventional terrestrial surveying by wading with a pole, multi-media photogrammetry, or spectrally based depth retrieval. The challenge for laser based approaches in such very shallow waters $(<2 \mathrm{~m})$ is the difficulty of discriminating between echoes from the surface and the bottom. This work presents an algorithm for the detection of surface, volume, and bottom (SVB) designed to meet this challenge while requiring only a single wavelength $(532 \mathrm{~nm})$ sensor. The accuracy of the algorithm is cross validated against reference measurements obtained from terrestrial survey with a total station and shows negligible bias and virtually no depth dependence for the experimental dataset.
\end{abstract}

Keywords: LiDAR, River, Bathymetry, Shallow Water, Full Waveform

2010 MSC: 86A05

\section{Introduction}

Mapping of underwater topography is a routine task in hydraulic engineering. Detailed knowledge of the morphological structure of rivers, lakes, reservoirs, and coastal areas is a prerequisite to developing detailed models for flood planning and forecasting of response to catastrophic events. The additional consideration of ecological factors, as required by the EU Water Framework Directive, also requires cost effective monitoring protocols (European Union, 2000). Airborne Hydro Mapping (AHM) (Steinbacher and Aufleger, 2013; Mandlburger et al., 2011; Mandlburger, 2017) is a method to meet such demands. AHM is an application of airborne laser bathymetry (ALB) for precise remote sensing of shallow water bodies as encountered in rivers, lakes, riparian areas, and reservoirs, as well as shallow coastal areas.

ALB is an active remote sensing technique based on light detection and ranging (LiDAR), measuring the round trip time of a laser pulse travelling between the sensor, in air, and the target, in water. While it is a very important method, several alternatives to ALB exist such as passive optical remote sensing based on measurements of radiance reflected from a water body (Legleiter et al., 2004), multimedia digital photogrammetry (Maas, 2015; Westaway et al., 2001) acoustic echo sounding, or more traditional surveying by wading making use of real-time-kinematic (RTK) GNSS (Global Navigation Satellite System) or total station equipment. A direct comparison

\footnotetext{
${ }^{*}$ Corresponding author

Email address: rschwarz@riegl . com (R. Schwarz)
}

of the capabilities of hyperspectral imaging and ALB is made by Legleiter et al. (2015). For ALB they mention the ability to directly measure bed elevations, but report a difficulty to measure very shallow depths. Recently several additional interesting ideas appeared, aiming to circumvent the costly manual approach, such as the use of unmanned surface vehicles (USV) employing a mechanical sensing bar (Sahalan et al., 2016), or the use of a low cost structured light sensor such as the Microsoft Kinect (Klopfer et al., 2017).

Measuring bed elevations with ALB is a more complex problem than measuring terrain elevations with topographic LiDAR since two media, air and water, are involved. The laser beam propagates at a much slower speed within the water body (three quarters the speed of light in air) and changes its direction at the air-water-interface; both effects are described by Snell's law. Reconstruction of the water surface therefore is an inherent necessity for determining correct bed elevations. Several approaches have been documented: Pe'eri and Philpot (2007) investigated a two wavelength approach for shallow waters. While laser pulses at the near infrared (NIR) will be reflected from the surface mainly, laser pulses at the wavelength of green light are capable of penetrating into the water column and are eventually scattered back from the bottom. Another approach has been described by Mitchell and Thayer (2014), who make use of the polarization preservation effect of the water surface to discriminate between bottom and surface returns. The authors performed promising experiments in the laboratory, but it remains to be seen how the scheme will perform under real-world conditions in large-area airborne mapping scenarios. Another technique combining a laser range finder operating in the NIR
Sharing info: https://www.elsevier.com/about/policies/sharing. 
range with a green laser imaging system has been described by Shen et al. (2017). They use a camera to observe the displaced images of the green laser as seen when reflected from the surface and the bottom. Mandlburger et al. (2017) investigated the question of whether it is possible to reconstruct the water surface from redundant observations of the riverbed. They conclude with a positive answer given a couple of preconditions are satisfied, such as the presence of sufficient morphological details and the ability to capture water bottom topography from different directions.

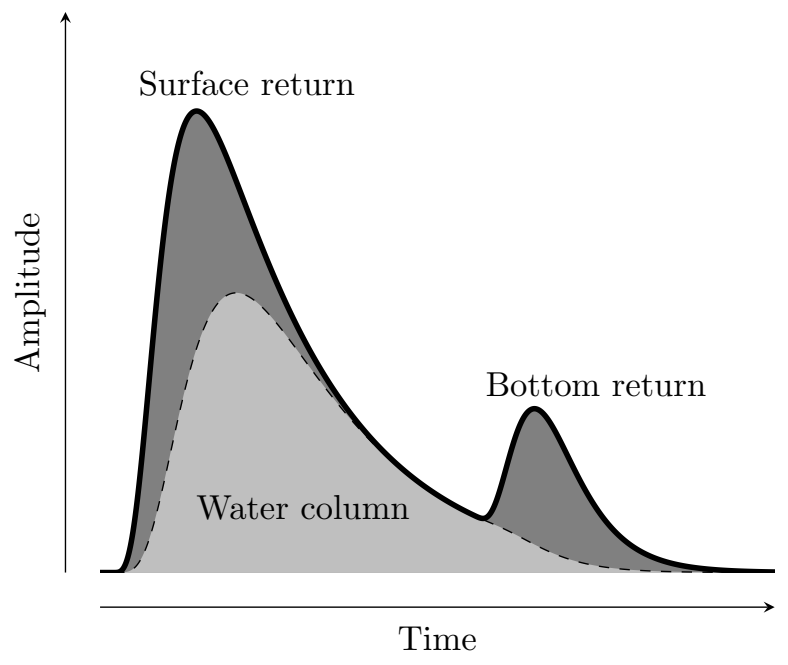

Figure 1: Schematic of three component green single wavelength received signal.

From a complexity point of view, simpler sensor concepts are favorable, such as those that employ a single wavelength laser only. To understand why shallow waters $(<2 \mathrm{~m})$ pose an extra challenge for single wavelength systems, one may consider that ALB inherently is a multi-target method because of the need to detect both, surface and bottom. Since target discrimination for such a single channel system is limited by laser pulse duration and receiver bandwidth (Pfennigbauer et al., 2009), a lower bound for the sensor's ability to measure shallow waters is to be expected. Given this limitation, it is an open question as to whether a standard multi-target capable sensor can be used for bathymetry. Mandlburger et al. (2013) consider this question based on a RIEGL VQ-820-G $(\lambda=532 \mathrm{~nm})$ sensor. They find that the online waveform processing (OWP) algorithm (Pfennigbauer et al., 2009), implemented on the sensor to deliver points in real time, results in a fuzzy cloud of interface echoes with its maximum density occurring approximately $10 \mathrm{~cm}$ to $25 \mathrm{~cm}$ below the surface. In contrast, the echo points from the bottom show a low spread in vertical direction. It is well known that the echo return from a water body typically consists of three contributions (Fig. 1): a return from the air-water interface (i.e. the water surface), a return from the water column, and a return from the water bed (e.g. Guenther et al., 2001). Within the water column, back-scattering by dissolved organic particles with varying density causes quasi-random intermediate peaks in the recorded return signal resulting in a large number of fuzzy points extending below the surface. Mandlburger et al. (2013) show that it is possible to approximate the reference water surface level via statistical analysis of these responses.

When it became clear that relatively simplistic detection and estimation schemes based on a threshold alone, would not allow one to infer more information from the return signals, sensors were built that employed digital signal processing to operate on the digitized full waveforms received. This type of sensor can also store relevant parts of the received signals for off-line data processing, which spurred the development of full-waveform analysis (FWA). The objective of any such FWA algorithm is to infer the reasons for the specific form of the received waveform. Most of these algorithms implicitly assume that the superposition principle (i.e. linearity) is valid for the echoes returned from different scatterers. However, since it is a demanding engineering task to build receivers and digitizers, that maintain a high degree of linearity over a wide dynamic range of input signals (e.g. weak bottom and strong surface), combined with high operational speed, non-linearity cannot be avoided entirely. Consequently, a few of these algorithms consider nonlinear receiver behavior explicitly (Pfennigbauer and Ullrich, 2010; Hartzell et al., 2015), some at least mention presence of non-linearity in the receiver (Zhao et al., 2018; Fuchs and Tuell, 2010), but often the issue of linearity is not addressed explicitly. Collin et al. (2008), however, were aware of the non-linear, logarithmic receiver behavior because they suggested that a linear fit of the received response could be used to estimate the attenuation coefficient. Abady et al. (2014) employ linear segments to approximate the water column, although they do not discuss receiver linearity.

At this point, we want to emphasize that linearity is of particular importance when examining distributed targets such as the water column. Wagner et al. (2006) show that the receiver output can be understood as a convolution, thus implying linearity, between the system waveform and the differential back-scatter cross-section (dBSC), but they do not make use of the true system waveform. Cawse-Nicholson et al. (2014) suggest using echoes from hard targets, which are essentially equivalent to the system waveform, for deconvolution. Schwarz et al. (2017) went one step further and proposed an exponential decomposition algorithm for bathymetric waveforms making use of an explicit recording of the system waveform. In that work the authors used an unlimited chain of multiple exponential segments as a model for the water surface, volume, and bottom. One of the key features of their algorithm is that their model of the system waveform (see also: Schwarz and Pfennigbauer, 2018) makes possible a closed form expression of the convolution of exponential dBCS models with the system waveform, a key requirement to efficient implementation of non-linear parameter fits. They demonstrated the feasibility and effectiveness of their method, but due to a lack of reference data they did not sufficiently assess the accuracy of the method.

In the present work we extend the work of Schwarz et al. (2017) by presenting a surface-volume-bottom (SVB) algorithm based on the aforementioned exponential decomposition, but restrict the unlimited chain of exponentials to a more compact model of 10 unknown parameters with explicit provision for the surface and bottom layers. We examine questions 
regarding (a) the appropriateness and quality of the SVB algorithm, (b) the accuracy of the riverbed estimate, and (c) the degree to which it is possible to approach the theoretical limit for target discrimination in very shallow waters. We address these questions by empirical evaluation of the algorithm and comparison of ALB data with terrestrially surveyed reference points.

\section{The SVB Algorithm}

\subsection{Design of the Algorithm}

For cases of practical relevance the output signal of the receiver of a LiDAR system can be written as a convolution integral

$$
p(t)=\int_{-\infty}^{+\infty} h(t-\tau) \sigma(\tau) d \tau=h(t) * \sigma(t)
$$

with $\sigma(t)$ the differential backscatter cross-section (dBCS) and the system waveform $h(t)$ (see e.g. Wagner et al., 2006). The system waveform $h(t)$ is equal to the received signal when pointing the sensor in a direction perpendicular to a flat extended target since in this case the dBCS equals Dirac's delta function (Pfennigbauer et al., 2013; Cawse-Nicholson et al., 2014; Hartzell et al., 2015). Dirac's delta function, although strictly speaking not a real function, is a mathematical object of zero width and infinite height while the area of the function remains a finite number. These properties make the delta function an adequate model for representation of a discrete scatter event, where the area of the function corresponds to the amount of reflected energy.

The SVB algorithm essentially minimizes the squared sum of differences

$$
\mathcal{E}(\varphi)=\sum_{n=1}^{N}\left(p_{m}\left(t_{n}, \varphi\right)-p\left(t_{n}\right)\right)^{2}
$$

between a continuous model of the received signal $p_{m}(t, \varphi)$, sampled at $t=t_{n}$, and the waveform samples $p\left(t_{n}\right)$ by finding the optimum parameter vector $\varphi=\varphi_{\text {opt }}$ where $t_{n}$ are the sampling time instances of the sample set of size $N$. The model $p_{m}$ is defined by the convolution

$$
p_{m}(t, \varphi)=h_{m}(t) * \sigma_{m}(t, \varphi)
$$

of the two sub models $h_{m}$ for the system waveform and $\sigma_{m}$ for the dBCS.

A basic model of the dBCS for a water body can be given as two Dirac's delta functions, one for the surface, and one for the bottom, plus one exponential function segment for the water column (Guenther, 1985). However, this model is only appropriate for laser beams hitting both the water surface and bottom at zero-degree incidence angle. For a non-zero angle of incidence the returned pulse is subject to broadening and is not described properly by a Dirac's delta function. Typical ALB systems employ conical scanning (i.e. Palmer scanner) with typical off-nadir angles of approximately $20^{\circ}$. Thus, neither the surface nor the bottom fulfill the zero-degree incidence angle requirement and therefore require a different dBCS model. Pfennigbauer et al. (2013) show that pulse broadening can be described by a convolution of a Gaussian-shaped system waveform with the lateral intensity shape of a Gaussian-shaped beam, resulting in another Gaussian-shaped, but wider, pulse. For reasons of mathematical tractability, we use a simplified model to describe the broadening effect, namely the convolution of a boxcar shaped function with the model $h_{m}$ of the system waveform.

\subsection{Implementation Details of the Algorithm}

For an efficient implementation of the minimization explicit knowledge of the partial derivatives of $\mathcal{E}(\varphi)$ is desirable. For this reason, we make use of a model of $h_{m}(t)$ defined as the real part of a sum of exponentials

$$
h_{m}(t)=\mathbb{R e}\left\{t_{+}^{0} \sum_{i=1}^{I} \alpha_{i} e^{\beta_{i} t}\right\}
$$

where $\alpha_{i}$ and $\beta_{i}$ are the complex valued model parameters, $I$ is the model order (the number of components), and $t_{+}^{0}$ is the Heaviside step function which is 0 for $t<0$ and 1 elsewhere. The parameters $\alpha_{i}$ and $\beta_{i}$ are determined by a parameter fit to match the model $h_{m}$ with a recording of the system waveform and are stored in the sensor as part of the calibration data (Schwarz et al., 2017). In Fig. 2, a typical model of the system waveform of a RIEGL VQ-880-G topobathymetric airborne laser scanner can be seen.

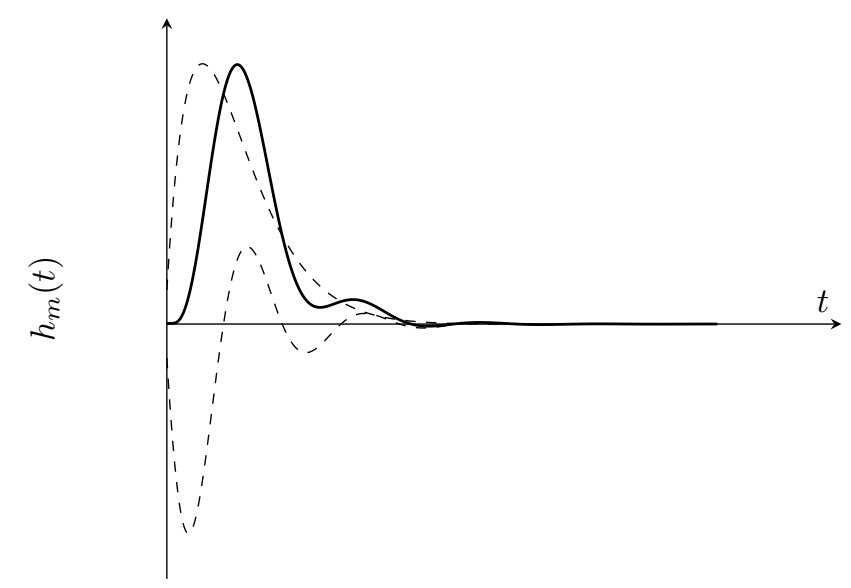

Figure 2: Exponential components (dashed) and sum of components of the sensor's system waveform model (solid) as a function of time.

Fig. 3, displays the refined SVB model of the dBCS where the two Dirac's delta function elements have been replaced by boxcar shaped functions and a second exponential has been added. The boxcars represent the pulse broadening effect and the second exponential is allowed to extend below the bottom to take care of late echoes, likely caused by multiple scattering. Near the surface and near the bottom the echoes from the interface layers and the column are overlapping. This is modeled by the analogous overlap of boxcar and exponential segments. The exponential decay of the $\mathrm{dBCS}$ is described by the parameter $\gamma$ which is restricted to be the same for both exponentials. 


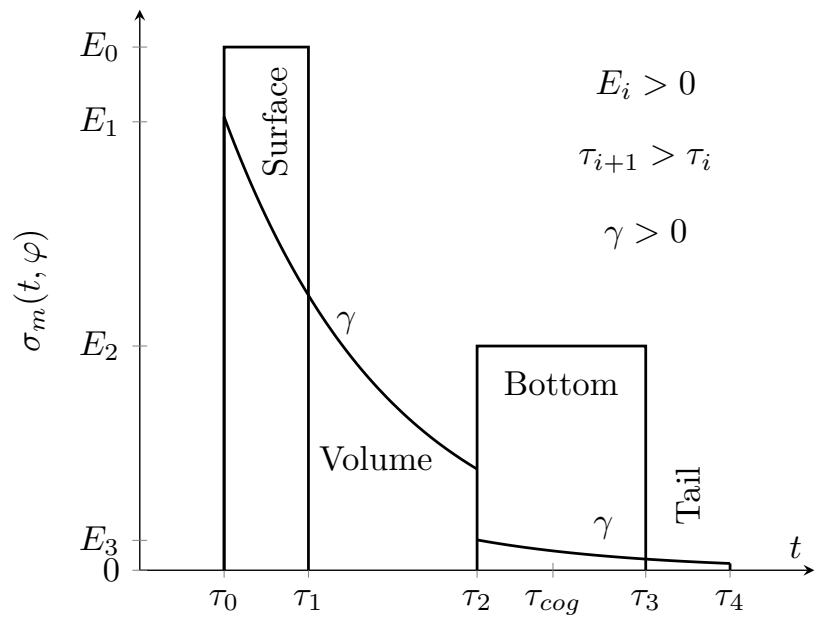

Figure 3: SVB model with 10 parameters $E_{0} \ldots E_{3}, \tau_{0} \ldots \tau_{4}$ and $\gamma$. Surface and bottom are modelled by boxcars to account for beam spreading caused by off nadir incidence. Effects from the water volume are modelled by two exponential segments. The tail below the bottom takes care of late echoes, likely caused by multiple scattering. $\tau_{c o g}$ is the center of gravity of the bottom and tail segments with respect to $\tau_{2}$.

The 10 elements of the parameter vector

$$
\varphi=\left(E_{0}, E_{1}, E_{2}, E_{3}, \tau_{0}, \tau_{1}, \tau_{2}, \tau_{3}, \tau_{4}, \gamma\right)
$$

are bound by the inequalities:

$$
\begin{array}{rlrl}
E_{i} & >0 & & i=0 \ldots 3 \\
\tau_{j+1} & >\tau_{j} & j=0 \ldots 3 \\
\gamma & >0 & &
\end{array}
$$

Since the parameter optimization will not reliably converge from random start values, $\tau_{0}$ and $\tau_{2}$ must be initialized with values close to surface and bottom positions. The algorithm starts by identifying the two peaks of highest significance at position $t_{m s}$ for the most significant and $t_{s s}$ for the second most significant peak. Instead of using a simple threshold trigger to find these locations a method inspired by topographic considerations (Kirmse and de Ferranti, 2017) has been used. First for every sample point $P_{i}=\left(t_{i}, p\left(t_{i}\right)\right)$ isolation and prominence are calculated. (An optimization is possible by considering only points of local maxima.) Isolation of a point $P$ at some amplitude is defined as the smallest distance to a point at the same amplitude when connecting the points by a straight line that is entirely contained in the upper half of the graph (Fig. 4). Prominence is defined as the difference between the amplitude of the point $P$ and the absolute minimum of the segment of the graph constituted by the isolation line. The significance of a point $P$ then is defined as the product of prominence, isolation, and amplitude. Finally from the positions of the two most significant peaks the initialization of $\tau_{0}$ and $\tau_{2}$ is calculated: Considering that the convolution of a function $f(t)$ (e.g. the dBCS model) with another function $g(t)$ (e.g. the system wave) will shift the center of gravity $(C O G)$ of the first function by the $C O G$ of the second,

$$
\operatorname{COG}(f(t) * g(t))=\operatorname{COG}(f(t))+\operatorname{COG}(g(t)),
$$

with the $C O G$ of a function $f(t)$ defined as

$$
C O G(f)=\frac{\int_{-\infty}^{\infty} \tau f(\tau) d \tau}{\int_{-\infty}^{\infty} f(\tau) d \tau}
$$

the $\operatorname{COG}\left(h_{m}\right)$ of the system wave is subtracted from the most significant positions and used to initialize $\tau_{0}$ and $\tau_{2}$.

$$
\tau_{0}=t_{m s}-\operatorname{COG}\left(h_{m}(t)\right), \quad \tau_{2}=t_{s s}-\operatorname{COG}\left(h_{m}(t)\right) .
$$

The above assumes that the peak positions of the waveform are close, at least approximately, to the $C O G$ defined by a surrounding of the peaks. The widths of the boxcars $\tau_{1}-\tau_{0}$ and $\tau_{3}-\tau_{2}$ are initially set equal to the width of the system waveform. The width of the second exponential $\tau_{4}-\tau_{2}$ is initially equal to $\tau_{3}-\tau_{2}$. The sample values at the both most significant positions are used to initialize the amplitudes $E_{i}$ of the boxcars and the exponentials:

$$
E_{0}=E_{1}=0.5 p\left(t_{m s}\right), \quad E_{2}=E_{3}=0.5 p\left(t_{s s}\right) .
$$

The damping $\gamma$ is initialized with

$$
\gamma=\frac{1}{\tau_{2}-\tau_{0}} .
$$

Since, in the case of very shallow waters, the second most significant peak might not represent an interface layer (surface or bottom), due to severe overlapping of the echo waveforms, other alternative start values in addition to the most prominent peak are used. These alternatives are 1) the third prominent peak, 2) a position $t_{z c}$ defined by the first zero crossing before the most prominent peak, and 3) a position $t_{c o g}$ offset by the $C O G$ of the system wave in the front of $t_{m s}$. All four sets of start parameters are tried and the fit with the lowest residual error is taken as the result.

Fig. 5 shows the result of a parameter fit. The thick black line is the result $p_{m}(t, \varphi)$ of the convolution of the dBCS, drawn in red, and the system waveform. For comparison, the OWP echo locations are drawn in green. These locations, which represent the round trip times between the sensor and the targets, are drawn at the positions of the waveform peaks. However, this is only by convention, the locations could have been drawn at the fronts of the waves or at points on their leading edges as well. For a ranging unit that is based on peak detection, the convention for visualization of the ranging results is to identify the target locations with the positions of the peaks. The visualization of the OWP, a correlation based algorithm, adheres to this convention by applying a constant offset to align the ranging results with the peak positions. Now the dBCS, drawn in red, follows a similar convention: Since the thick black line is the result of a convolution of the system waveform and the $\mathrm{dBCS}$ it cannot be nonzero where the $\mathrm{dBCS}$ is zero. On the other hand we need to align the front $\tau_{0}$ with the echo location given by the position of the OWP points to get the same range. This is done by applying a sensor dependent offset which has to be determined once during sensor calibration.

Finally the SVB parameters require a mapping to the water surface and the bottom points. From the set of parameters $\tau_{0}$ is 


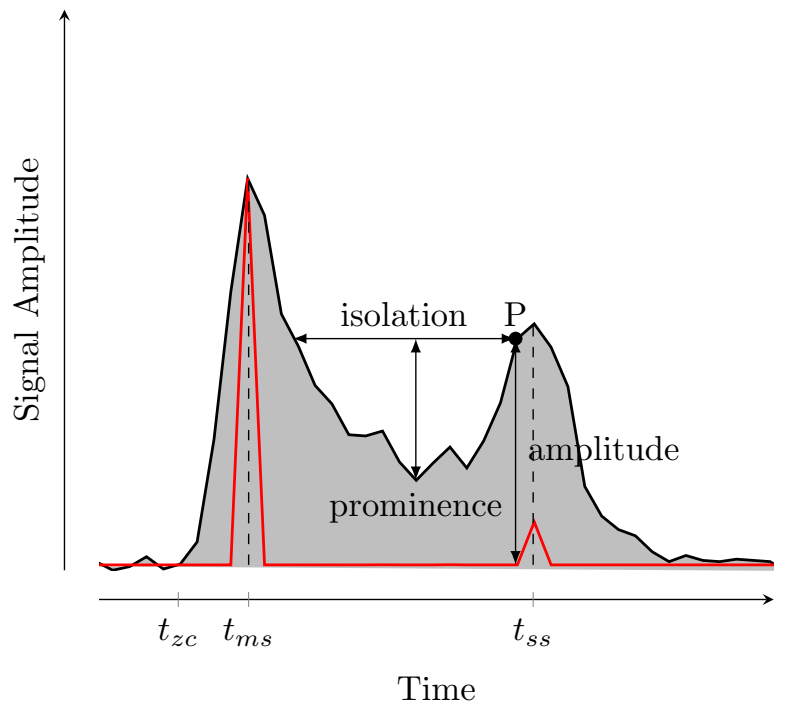

Figure 4: Isolation, prominence, and amplitude of a point $P$. Significance of $P$ then is the product of isolation, prominence, and amplitude. Black line: waveform data; red line: calculated significance rescaled to the maximum of the data for better display. The most significant peak is at location $t_{m s}$, the second most is at $t_{s s}$, and the closest zero crossing before $t_{m s}$ is at $t_{z c}$.

used to give the distance to the water-surface and $\tau_{2}+0.5 \tau_{\operatorname{cog}}-\tau_{0}$ is used to give the (uncorrected) length of the laser beam in water. Here $\tau_{\operatorname{cog}}$ is the $C O G$ of the second boxcar and exponential. The given mapping has been determined by experiment and has been chosen because it minimizes the systematic errors.

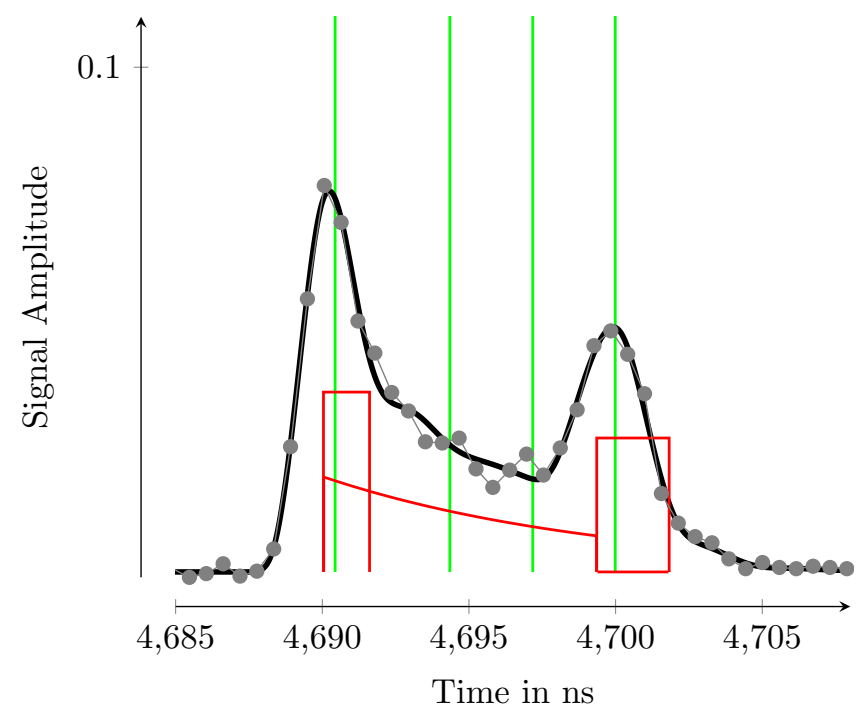

Figure 5: Received signal; sensor height: about $704 \mathrm{~m}$; water depth: about $1 \mathrm{~m}$; gray dots: waveform samples; green: OWP position results; red: fitted SVB model; black solid: SVB model convolved with system waveform.

\section{Study Area and Datasets}

The Pielach River (Fig. 6) is a medium-sized, gravel bed tributary of the Danube River with a gradient of $0.2 \%$ within

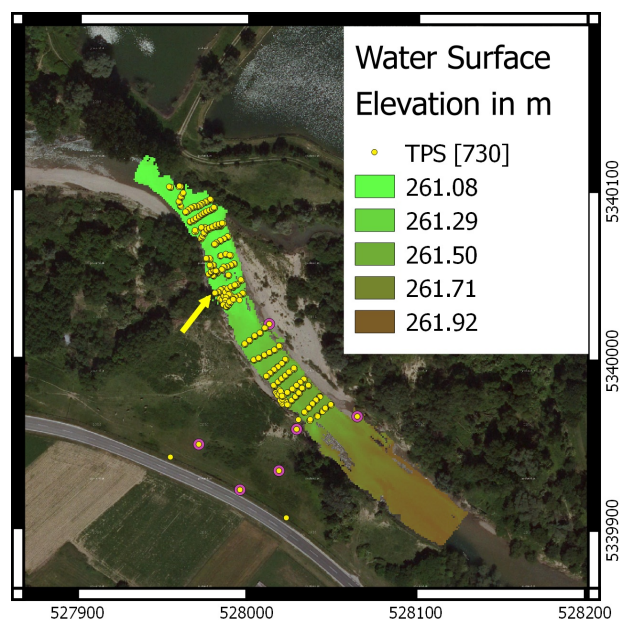

Figure 6: Study area Neubacher Au (Pielach River, Austria); orthophoto: basemap.at; water surface: raster map with color coded water level heights; TPS: GNSS and tachymetric reference points. The arrow points to the profile shown in Fig. 13.

the study area, located in the Neubacher $\mathrm{Au}$ in Lower Austria $\left(48^{\circ} 12^{\prime} 50^{\prime \prime} \mathrm{N}, 15^{\circ} 22^{\prime} 30^{\prime \prime} \mathrm{E}\right.$; WGS 84) about $100 \mathrm{~km}$ west of Vienna, the capital city of Austria. The area is routinely used for instrument calibration flights by the sensor manufacturer RIEGL and has been repeatedly used as a site for scientific studies. For more details about the site see Mandlburger et al. (2015). Data acquisition for the study at hand was carried out in the course of a RIEGL VQ-880-G-IR sensor calibration flight on June, 16, 2016 (full leaf-on). For validation purposes 730 checkpoints were simultaneously measured by means of conventional surveying with a total station, while wading with a pole. Position and orientation of the total station via free stationing was based on a local reference point network measured by RTK GNSS.

The VQ-880-G-IR is a dual wavelength sensor operating with a $532 \mathrm{~nm}$ green and $1064 \mathrm{~nm}$ NIR (near infra-red) laser with a nominal pulse repetition rate of $500 \mathrm{kHz}$. The per pulse energy of the green laser is $10 \mu \mathrm{J}$ and the pulse width is $2 \mathrm{~ns}$. The pulse returns are amplified in two independent parallel receiving channels of different gain, each sampled at a rate of $1.5 \mathrm{GHz}$ with a depth of 12 bits. Depending on trigger conditions, the waveform data are evaluated on-line and additionally stored to disk for post processing. The scan mechanism for the green laser creates a circular pattern on the ground, yielding a constant $20^{\circ}$ off nadir angle, while the NIR laser echoes are aligned in parallel scan lines perpendicular to the flight direction. The beam divergence of the green laser was set to $1.1 \mathrm{mrad}$ resulting in a footprint diameter of approximately $80 \mathrm{~cm}$ on the ground.

The area of interest is covered by 4 overlapping flight lines. For the green laser channel, the scan data of each flight strip are separated into a forward and backward looking semicircle, resulting in 8 distinct datasets. The effective point density over the study area making use of all four flight lines is about $60 \mathrm{~m}^{-2}$.

Since laser pulses in the infra-red are not penetrating below the water surface, these data points have been used as a refer- 
ence for evaluating the accuracy of estimates of the water surface elevation inferred from the green laser. The number of points returned from the water surface is largest when the flight trajectory is exactly over the river axis because NIR echoes from the water surface can only be detected at small off-nadir angles. The flight plan has been adjusted accordingly to maximize the usable laser echoes from the water surface yielding about $3 \mathrm{~m}^{-2}$ to $5 \mathrm{~m}^{-2}$.

\section{Methods}

Data processing was carried out using the sensor manufacturers software RiPROCESS (RIEGL, 2018). In addition to standard processing steps like multiple time-around resolution, direct georeferencing, strip adjustment, refraction correction, etc., the SVB algorithm was also implemented in RiPROCESS. Distance calculations and raster map creation was completed with CloudCompare (Girardeau-Montaut, 2018). The IR-Density map was created with the software package OPALS (Mandlburger et al., 2009). For all the following steps, only points and waveforms from the water body have been subjected to processing.

In order to asses the performance of the SVB algorithm, specific point cloud datasets have been generated. One consists of points from the SVB algorithm (i.e. the result of the full waveform analysis), the other consists of OWP points only. In the SVB set, the point at $\tau_{0}$ classifies as surface and the point at $\tau_{2}+0.5 \tau_{\text {cog }}$ classifies as bottom (see Fig. 3 ). For the OWP-set, points were classified into surface and bottom using the following ad-hoc algorithm: OWP multi-target points (i.e., multiple points from the same laser shot) have been grouped into first, intermediate, and last targets. The first targets were then classified as surface and the last targets classified as bottom of the water body. Manual data cleaning was employed to remove outliers like first echoes in the riparian vegetation or last echoes below the water bottom. The SVB and OWP point clouds were independently processed.

First, separate water surface models were calculated from the respective points of the OWP and SVB dataset and used for refraction correction of the bottom points by means of RiHydro, a software module of RiPROCESS.

Next, triangle meshes were generated for the set of water surface points and the set of bottom points using the Delauny 2.5D option of CloudCompare. Finally, for each terrestrially surveyed reference point the signed distance to the respective bottom mesh was calculated. Analogous processing steps were applied to the water surface reference points from the NIR laser channel and the water surface meshes.

Additionally, for the point cloud of the SVB bottom points statistical information about the SVB algorithm output was evaluated for each point: the Pearson correlation coefficient

$$
r=\frac{\sum_{n}\left(x_{n}-\bar{x}\right)\left(y_{n}-\bar{y}\right)}{\sqrt{\sum_{n}\left(x_{n}-\bar{x}\right)^{2}} \sqrt{\sum_{n}\left(y_{n}-\bar{y}\right)^{2}}}
$$

between the measured waveform and the model, the root mean squared error (RMSE)

$$
R M S E=\sqrt{\frac{\left(\sum_{n}\left(y_{n}-x_{n}\right)^{2}\right)}{N}}
$$

between waveform and model, and $\gamma$ the decay parameter. In Eq. 12 and Eq. 13, $y_{n}$ are the data samples and $x_{n}$ are the samples of the model for $n=1 \ldots N$, with $N$ the number of the samples of the waveform.

For assessing any dependency on depth, a depth measurent was derived for each SVB bottom point as the smallest distance of the point to the SVB surface mesh.

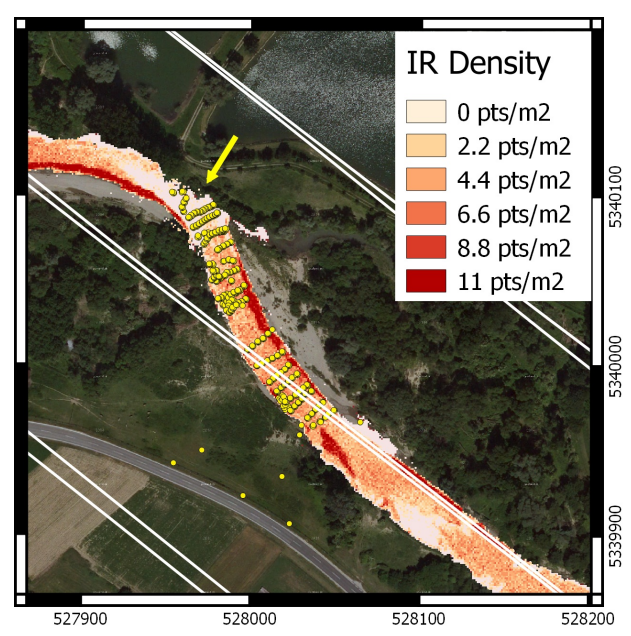

Figure 7: Density map of the infrared channel. The yellow arrow marks an area of poor NIR coverage of the water surface due to the larger incidence angle of the laser beam. Unfortunately this also is where the profiles of larger depths are located. White lines: sensor trajectories; orthophoto: basemap.at

The reference points for the water surface ideally would have been used as a (continuous) water surface model derived from the air-water interface echoes of the NIR laser channel. Although the NIR water surface point density was about $3 \mathrm{~m}^{-2}$ to $5 \mathrm{~m}^{-2}$ for most parts of the study reach, poor coverage of the areas where the profiles of larger depths were located prevented the interpolation of an accurate water surface model for the entire river section, see Fig. 7. It is worth mentioning that the observed void is caused by the larger incidence angle of the infrared beam and not by the larger water depths. Instead of a true NIR water surface model, we calculated the mean difference of the available NIR points to the respective surface meshes and used them as an offset between the SVB and OWP surface estimates and the NIR surface.

\section{Results and Discussion}

\subsection{Performance of SVB Algorithm}

The most important attribute of any full waveform algorithm is the accuracy of its results, such as ranging and intensity of the reflected signal. However, if the algorithm makes use of an explicit model as in our case, good agreement of the model and the measured data is important as well. For this reason, we evaluated the Pearson correlation coefficient and the root 
mean squared error between the recorded data samples and the modeled waveforms.

In Fig. 8 the results of the SVB decomposition are presented. Panel (a) shows a visible depth dependence of the Pearson $r$ coefficient but at a high degree of correlation of larger than 0.99 . Fig. 8 panel (c) shows the RMSE which does not exhibit depth dependence. The histogram of $r$ in Fig. 8 (b) highlights the high correlation between the model and the data and Fig. 8 (d) gives the mean value of the distribution of the $R M S E$ as $\mu=7.7$ digitization units, which is less than $0.2 \%$ of the range of the 12-bit digitizer.

The histogram of the decay parameter $\gamma$ as seen in Fig. 8 (f) is highly peaked at zero and there is also a peak near 120 . The first peak near zero indicates presence of a large number of exponentials which are almost shaped like a boxcar, meaning the exponential segment between $\tau_{0}$ and $\tau_{2}$ (and between $\tau_{2}$ and $\tau_{4}$ ) in Fig. 3 becomes

$$
\sigma_{m}(t)=\left.\left(\left(t-\tau_{0}\right)_{+}^{0}-\left(t-\tau_{2}\right)_{+}^{0}\right) E_{1} e^{-\gamma\left(t-\tau_{0}\right)}\right|_{\gamma=0} .
$$

Considering that a high value of the decay parameter makes an exponential look more like a Dirac delta function, the second peak of the distribution of Fig. 8 (f) indicates the presence of numerous Dirac delta-like exponentials. The numerical value of 120 bears no significance beyond being large. The value is related to an (arbitrarily chosen) upper bound that has been forced on the $\gamma$ parameter during the optimization step to avoid runaway. This has been validated by changing the upper bound and observing a corresponding change of the position of the second peak. The presence of the both peaks suggests a modification of the model by changing the $\gamma$ near zero to real boxcars and the $\gamma$ near 120 to real Dirac delta functions and exclude them from the statistics. The suggested changes to the model might be worth considering because $\gamma$ could be used for the estimation of turbidity. However for the present study this approach was not pursued.

The SVB algorithm will always attempt to identify exactly two boundary layers and characterize the medium in between. Looking at it in another way, this means that if the bottom echo is not strong enough the algorithm may infer the presence of some targets below the surface which are not the bottom but will erroneously be classified as such. We expect that a better understanding of the back-scatter behavior of the water column will allow an extension of the SVB algorithm to also reliably work in a SV (surface volume) mode. However, in the present dataset this issue was not of concern, because the good visibility conditions allowed to see the bottom through the water for the entire study reach.

Fig. 9 and 10 show the spatial distribution of a few parameters to further illustrate the performance of the algorithm. Fig. 9 compares the surface coverage of the detected water surface points of the SVB with respect to the OWP algorithm. The very shallow areas near the river banks while covered by surface points derived by the SVB algorithm are not available from the OWP algorithm. Fig. 10 is a map of the waterdepths derived by the SVB algorithm. Such depth distribution maps are not only of interest for building hydrological and erosion models but might also be of relevance for the biology of rivers (e.g., Maddock, 1999).

\subsection{Accuracy of Depth Estimation}

Depth is defined as the difference of the elevations of the water surface and the water bottom. As was expected, the water surface levels estimated by the SVB and OWP are slightly different with respect to the NIR surface. The SVB surface is $2.9 \mathrm{~cm}$ and the OWP surface $14 \mathrm{~cm}$ below the NIR surface, as can be seen by the dashed lines of Fig. 11 (b) and (d).

In the OWP case, the comparison of bottom points against the ground truth exhibits the existence of a small bias which is depth dependent (Fig. $11 \mathrm{~b}$ ) at a ratio of $3.6 \mathrm{~cm} \mathrm{~m}^{-1}$. For the SVB case there is virtually no dependence on depth (Fig. $11 \mathrm{~d}$ ). Elimination of this depth dependence is the result of the choice of the bottom point as $\tau_{2}+0.5 \tau_{\operatorname{cog}}$. This result, although encouraging, should be interpreted with caution since we cannot give a theoretical reasoning for the factor of 0.5 . On the other hand the correlation coefficient for the regression is only about 0.2 for both methods, so depth dependence is not very prominent anyways.

The statistics of the bottom elevation error (Fig. $11 \mathrm{a}$ and c) both show negligible bias for the both cases of SVB and OWP. Variances of the OWP and SVB histogram are comparable in magnitude. The OWP histogram exhibits bi-modality, which may be explained by the distortion of the pulse positions due to overlapping of the pulses. The dependence on depth (Fig. $11 \mathrm{~b}$ ) displays a second cluster of errors around a depth of $0.5 \mathrm{~m}$, a depth where severe overlapping already is taking place, see Fig. 12 (a).

Validation of ALB for very shallow waters in the regime of one meter and below is not found very often in the literature. Allouis et al. (2010) addressed this question for water depths below two metres. They used a two wavelength sensor with $5 \mathrm{~mJ}$ pulse energy at a repetition rate of $1 \mathrm{kHz}$ and a footprint diameter of $1.5 \mathrm{~m}$, compared to a pulse energy of $10 \mu \mathrm{J}$ at a rate of $500 \mathrm{kHz}$ and a footprint diameter of $0.8 \mathrm{~m}$ in our case. They find a bias growth of about $40 \mathrm{~cm}$ per meter which is a factor of 40 greater than the SVB results. However, they note that improvements might be possible by implementing a deconvolution of the physical model of the receiver, taking into account the back-scatter of the water column, and implementing proper refraction correction. Our results seem to substantiate these presumptions. Kinzel et al. (2007) investigated a riverine environment and asserted that a specialized algorithm is necessary for shallow water processing. In a later work Kinzel et al. (2012) undertook a thorough analysis of ALB for three rivers by making use of the ALPS (Airborne Lidar Processing System) bathymetry algorithm. Similar to our findings they report no considerable dependence on depth of the residual elevation error but find a somewhat larger standard deviation.

Fig. 13 shows an example profile taken at the position indicated by the yellow arrow of Fig. 6 . The blue points marking the water surface are slightly below the red points, which delineate the reference surface defined by the NIR echoes. The green points, marking the reference points at the bottom, coincide well with the refracted bottom points in maroon. 


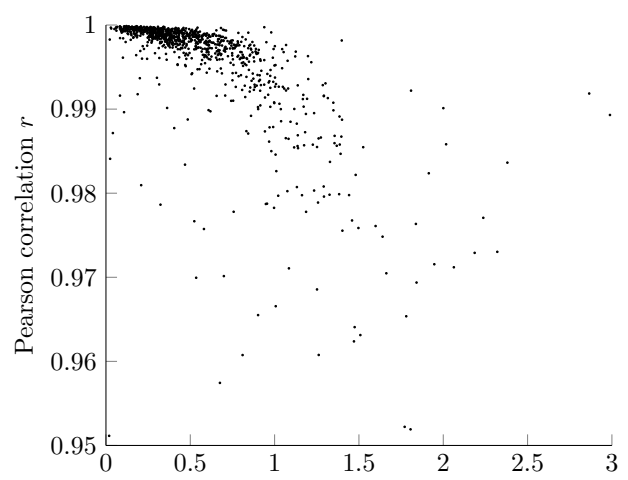

(a) Depth $h$ in $\mathrm{m}$

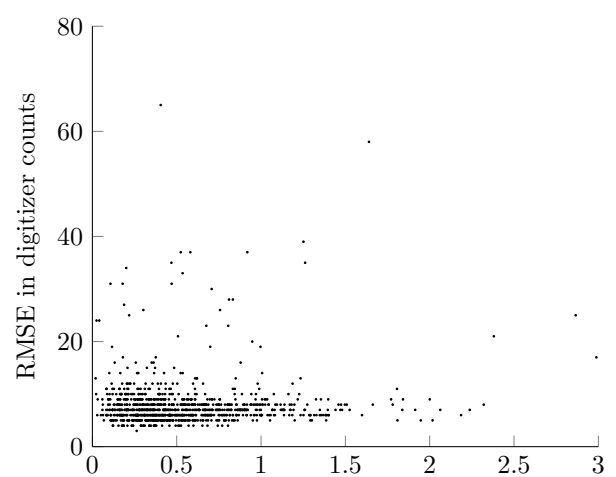

(c) Depth $h$ in m

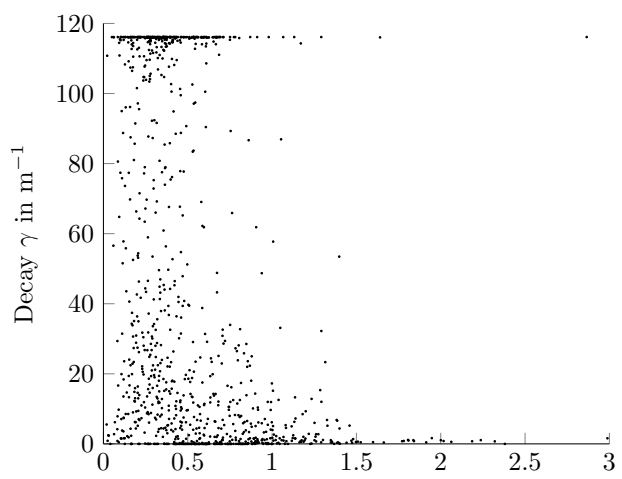

(e) Depth $h$ in m

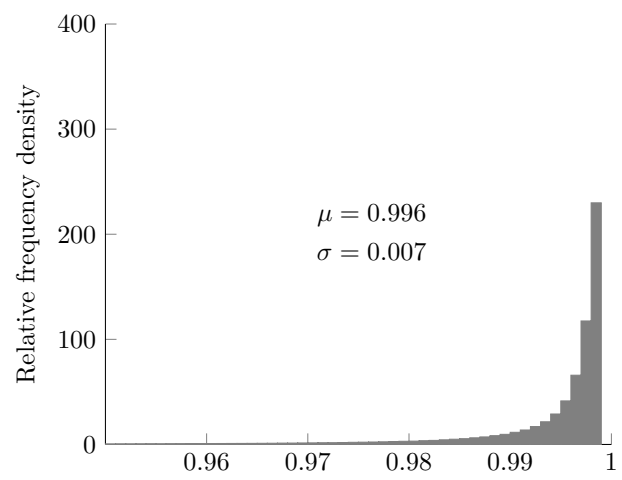

(b) Pearson correlation $r$

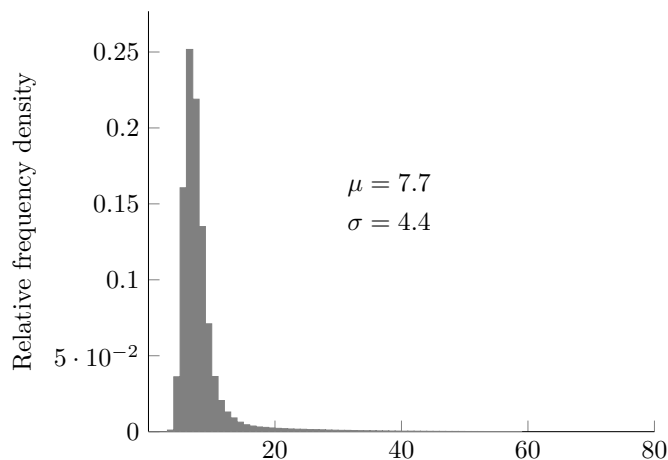

(d) RMSE in digitizer counts

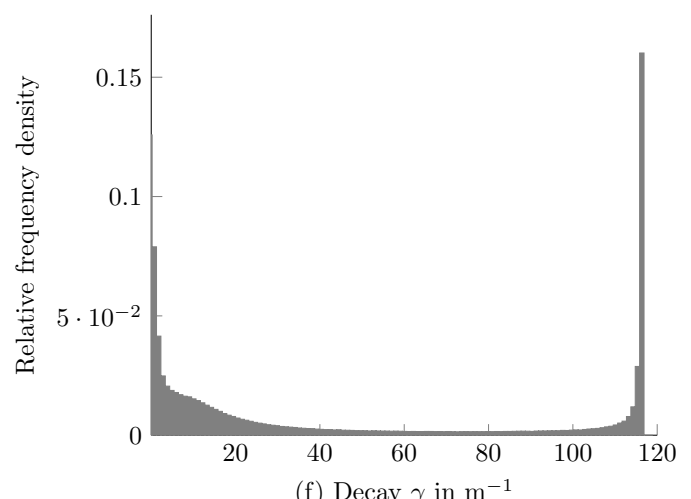

Figure 8: Statistics of SVB algorithm: (a) depth dependency of Pearson correlation coefficient $r$; (b) histogram of Pearson correlation coefficient $r$; (c) depth dependency of RMSE; (d) histogram of RMSE; (e) depth dependency of decay parameter; (f) histogram of decay parameter.

\subsection{Smallest Resolvable Depth}

The ability of a sensor to discriminate between two closely spaced targets is determined by the laser pulse width, the receiver bandwidth, and the sampling rate (Pfennigbauer et al., 2009). Legleiter et al. (2015) give a formula for the smallest resolvable depth

$$
\Delta h=\frac{1}{2} c_{w} \Delta t \cos \left(\theta_{w}\right)
$$

with $c_{w}$ the speed of light in water, $\Delta t$ the width of the system waveform, and $\theta_{w}$ the nadir angle of the laser beam. (The receivers bandwidth causes the width of the system waveform to be larger than the laser pulse width.) Assuming $\Delta t=2.81 \mathrm{ns,}$ $c_{w}=c_{0} / 1.33$, and $\theta_{w}=20^{\circ}, \Delta h$ evaluates to approximately
$30 \mathrm{~cm}$. However, equation (15) does not consider the effect of pulse broadening due to the off nadir incidence angle and footprint size of the laser beam. The results calculated by equation (15) therefore should be interpreted as an order of magnitude only.

Figure 12 displays two prototype waveforms from the very shallow regime. Fig. 12 (a) depicts the bottom at a depth of $0.5 \mathrm{~m}$ and Fig. 12 (b) the bottom at a depth of $0.25 \mathrm{~m}$. The green vertical bars mark the positions of the results of the OWP method. Although the trigger mechanism in the OWP case for closely spaced targets is able to resolve only one target (Fig. 12 b), the SVB modeling approach still is able to deliver two distinct target positions despite the substantial overlap of the pulses. It is worth mentioning that the SVB algorithm in 


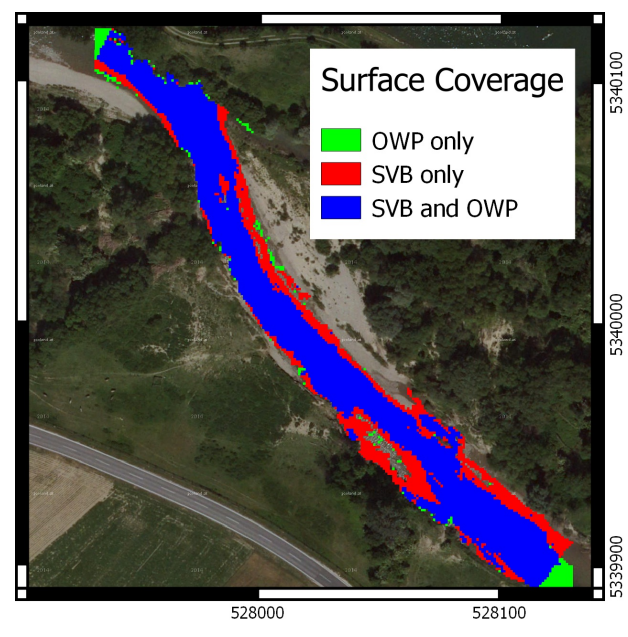

Figure 9: Surface coverage comparison of SVB and OWP algorithms: The SVB algorithm works even in the very shallow areas at the river banks; orthophoto: basemap.at.

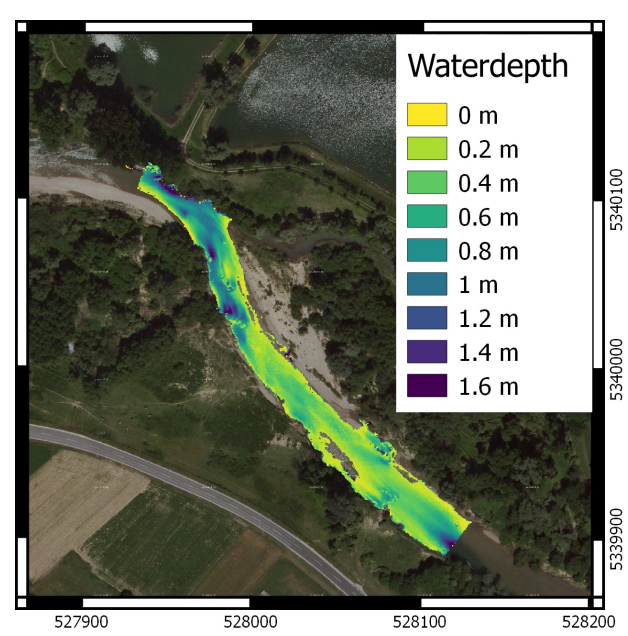

Figure 10: SVB Algorithm: Waterdepth map; orthophoto: basemap.at.

the case of shallow depths does not deliver exponential components. This is in contrast to Fig. 5 where at larger depths the exponential segment is properly modeled. Fig. 14 shows the profile from where the waveform in Fig. 12 (b) has been taken. The black line marks the laser beam direction of the waveform. The blue points of the surface as well as the maroon points from the bottom each describe a well defined layer. The points also can be seen to agree with the red and green reference points. The given example suggests that the SVB algorithm is able to resolve targets below the conventional limit given by Eq. 15 .

In an attempt to estimate the smallest resolvable depth we use the following reasoning: For a natural river it is plausible that the distribution of small depths will be continuous. This should be in contrast to regulated rivers where the steep riverbanks will cause a under representation of small depths. A sample distribution of depth values therefore should exhibit a continuous behavior towards zero in the case of a natural river.

Fig. 15 depicts such flow depth statistics, where panel (a) is made from OWP points, and panel (b) from SVB points. For the generation of these graphs an extended $2.0 \mathrm{~km}$ reach was used, surrounding the $400 \mathrm{~m}$ section covered by the reference points. The number $N$ gives the population size, after outliers (below zero and larger than $3 \mathrm{~m}$ ) have been removed. The shape of the histogram for the OWP method shows a lack of values for depths below $30 \mathrm{~cm}$. This lack of depth values between $0 \mathrm{~cm}$ to $50 \mathrm{~cm}$ for the OWP method also is visible in Fig. 11 (b), it is explained by the fact that the trigger mechanism of the OWP method needs to see a peak, and is consistent with the limit for the minimum resolvable depth of about $30 \mathrm{~cm}$ given by Eq. 15 . Kinzel et al. (2012) present a similar figure with the same lack of data for depths $<0.5 \mathrm{~m}$.

On the other hand, the shape of the flow-depth histogram generated from the SVB method exhibits a consistent approach towards zero. It is this continuous behavior for small values of the sample distribution that we interpret as an indication of the ability of the SVB method to resolve depths down to zero. This is a very encouraging result, but additional experiments will be necessary to explore the effects of parameters such as the reflectivity, structure, and roughness of the riverbed, as well as the influence of the state of the surface and optical properties of the water column, such as turbidity. In summary, the SVB method in the very shallow regime needs only a single waveform to deliver a water surface and a bottom point while the OWP method applied to the same waveform will only deliver the bottom point.

Smith et al. (2011) propose a gamma distribution model for the distribution of overland flow depths. Legleiter (2016) used empirical and theoretical models, the gamma function being a prominent representative of the latter, for calibrating the imageto-depth quantile transformation for inferring bathymtery form multi- or hyperspectral image data. It is interesting to see that the gamma distribution is a good fit for our data set as well. The red line in Fig. 15 (b) shows a two parameter gamma function obtained by a least squares parameter fit, which is in good agreement with the underlying sample depth distribution of the SVB method.

\section{Conclusions}

We have presented a new SVB (surface, volume, and bottom) algorithm for single wavelength airborne laser bathymetry and demonstrated that it is capable of inferring depth down to approximately one centimeter with very high fidelity. The algorithm is based on the method of exponential decomposition (Schwarz et al., 2017).

By comparison with GNSS reference points for the bottom, we showed that the resulting point cloud is an unbiased measure of river bed geometry with a variance of $3 \mathrm{~cm}$ with respect to the GNSS reference. Goodness of fit metrics comparing the measured and modeled waveforms (Pearson $r$ coefficient and the RMSE) do not exhibit depth dependence. Also the river bed elevation error does not exhibit depth dependence. Based on the given dataset we were able to show that the SVB algorithm is able to resolve depths smaller than predicted theoretically (Legleiter et al., 2015). 
(a)
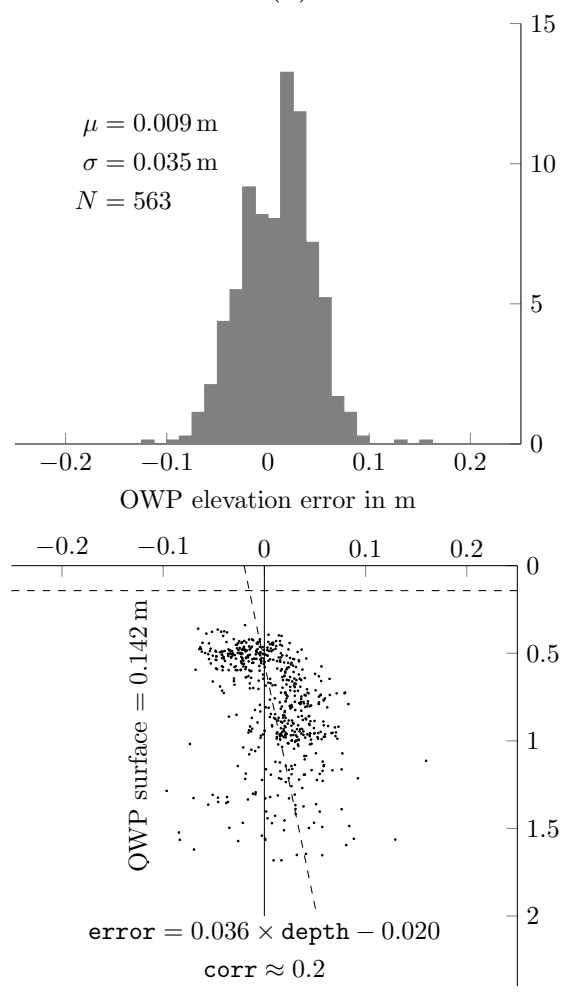

(b) (c)
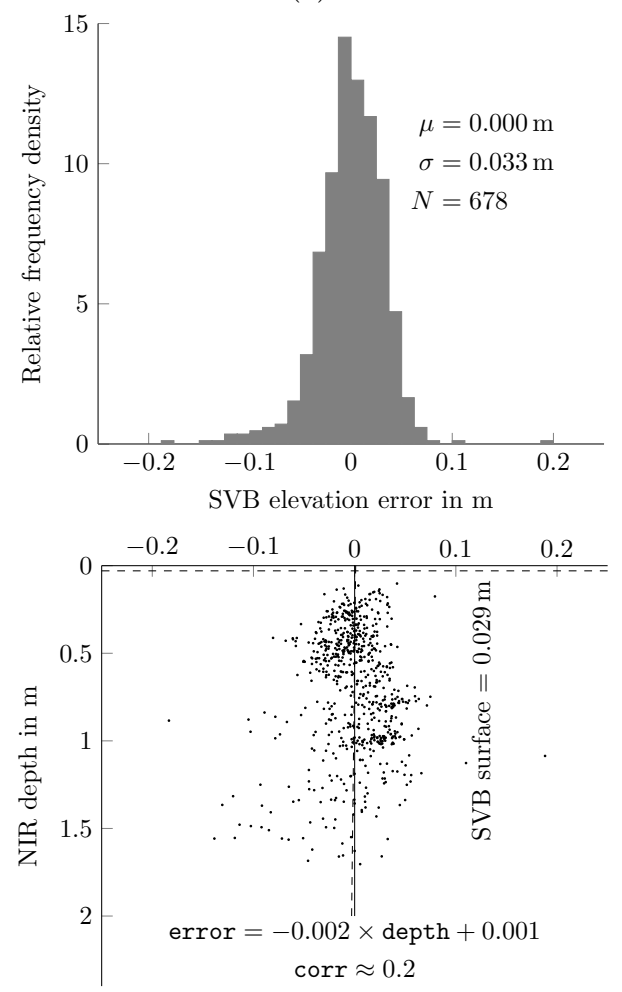

(d)

Figure 11: Statistics of elevation error. NIR depth has been offset corrected to reference the water surface defined by the NIR channel; the dashed horizontal lines indicate the mean values of the OWP (b) and SVB (d) surfaces. Panel (a) shows the OWP elevation error histogram, (b) shows the OWP depth dependent elevation error, i.e., OWP bottom minus reference, (c) shows the SVB elevation error histogram, and (d) shows the SVB depth dependent elevation error, i.e., SVB bottom minus reference.

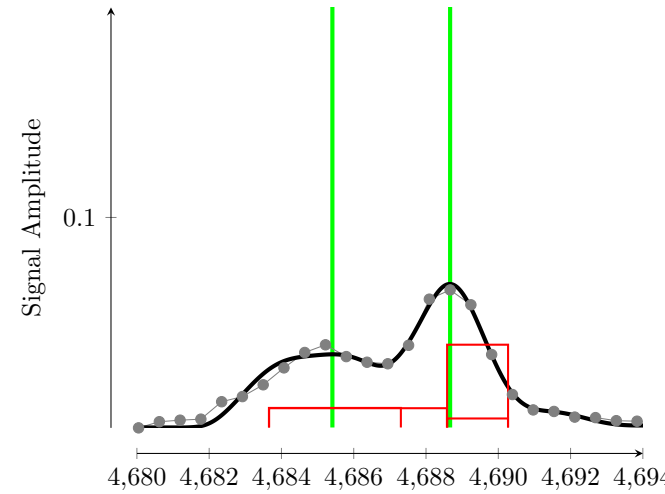

(a) Time in ns

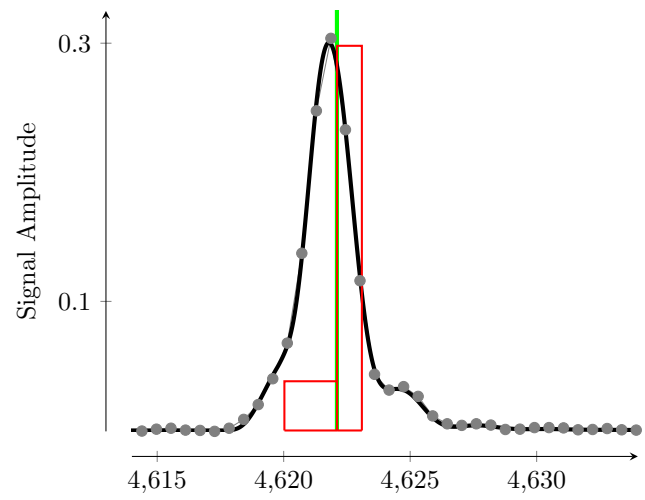

(b) Time in ns

Figure 12: Waveforms in very shallow water: (a) at a depth of $0.5 \mathrm{~m}$; (b) at a depth of $0.25 \mathrm{~m}$; both cases exhibit severe pulse overlapping. Dots: waveform samples; green: point positions detected by OWP method; red: the SVB model; black solid: SVB model convolved with system waveform.

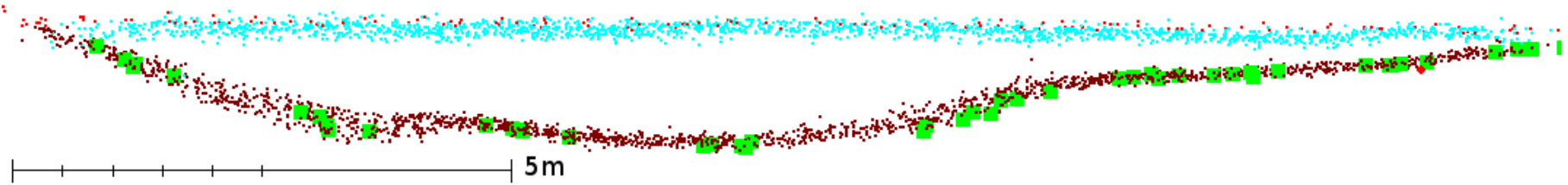

Figure 13: Example profile showing the water surface and refracted bottom points estimated by the SVB algorithm. Blue: the surface points; maroon: refracted bottom points; green: GNSS bottom reference points; red: NIR surface reference points. 


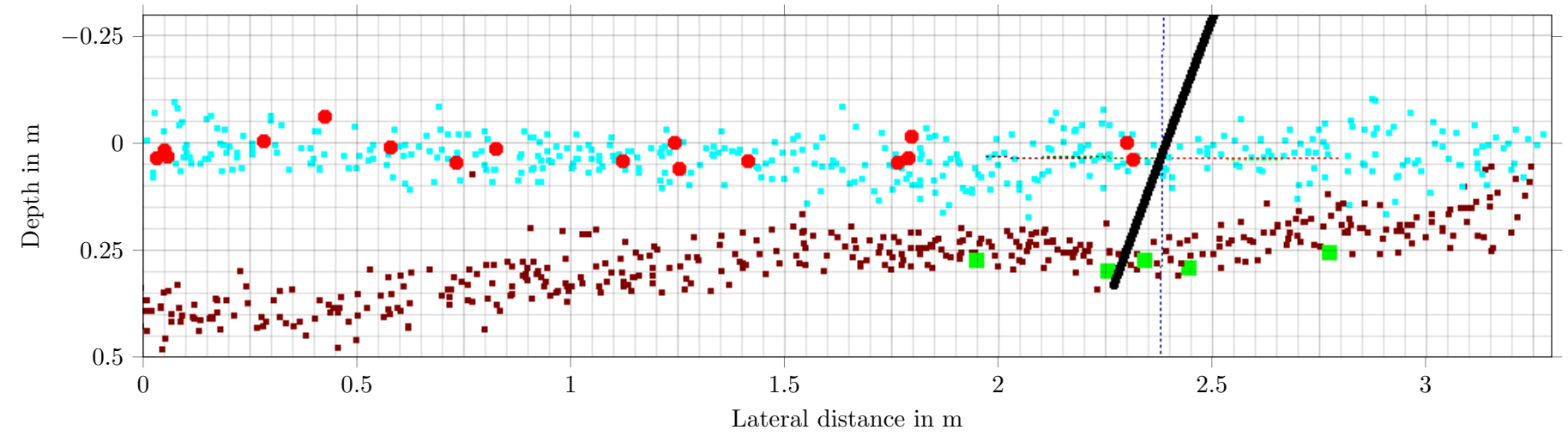

Figure 14: Example profile in very shallow water. The black line is marking the laser beam of the waveform shown in Fig. 12 (b). Blue: surface points; maroon: refracted bottom points; green: GNSS bottom reference points; red: NIR surface reference points. The width of the displayed segment is approximately $2 \mathrm{~m}$ in flow direction.

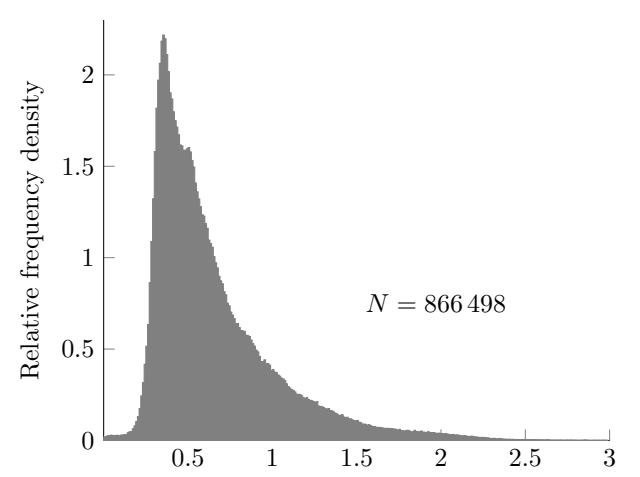

(a) Depth $h$ in $\mathrm{m}$

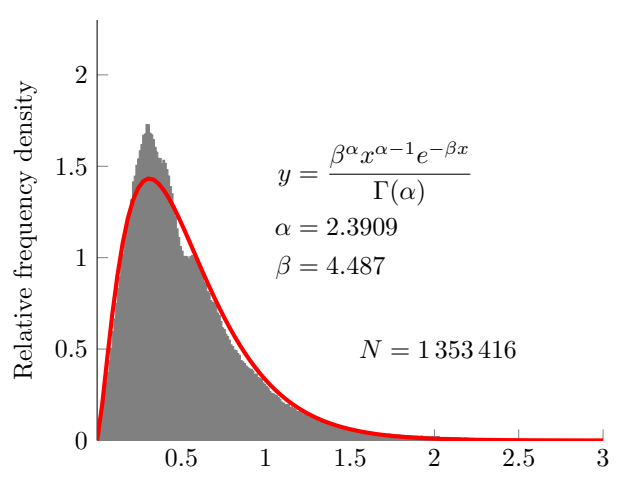

(b) Depth $h$ in $\mathrm{m}$

Figure 15: Histograms of flow depth $h$ after outlier removal $(0.0<h<3.0)$ : OWP method (a) and SVB method (b), red: fitted gamma distributions.

The decay parameter of the exponentially damped backscatter from the water column, although required for the modeling, was not further investigated in this study. This would have required waters of varying turbidity as well as water bodies of larger depths. Nevertheless a refinement of the SVB model is suggested, comprising the replacement of exponentials with boxcars or Dirac delta shaped functions in degenerate cases. The authors expect these refinements to be useful in future experiments with the prospect to use the decay parameter for turbidity assessment.

\section{Acknowledgments}

Acknowledgements: Gottfried Mandlburger's contribution to this paper was supported by the German Research Foundation (DFG) project 'Bathymetry by fusion of airborne laser scanning and multispectral aerial imagery'.

\section{References}

Abady, L., Bailly, J.S., Baghdadi, N., Pastol, Y., Abdallah, H., 2014. Assessment of Quadrilateral Fitting of the Water Column Contribution in Lidar Waveforms on Bathymetry Estimates. IEEE Geoscience and Remote Sensing Letters 11, 813-817. doi:10.1109/lgrs. 2013.2279271.
Allouis, T., Bailly, J.S., Pastol, Y., Roux, C.L., 2010. Comparison of LiDAR Waveform Processing Methods for Very Shallow Water Bathymetry Using Raman, Near-infrared and Green Signals. Earth Surface Processes and Landforms , 640-650doi:10.1002/esp. 1959.

Cawse-Nicholson, K., van Aardt, J., Hagstrom, S., Romanczyk, P., Schaaf, C., Strahler, A., Li, Z., Krause, K., 2014. Improving Waveform Lidar Processing toward Robust Deconvolution of Signals for Improved Structural Assessments, in: Turner, M.D., Kamerman, G.W., Wasiczko Thomas, L.M., Spillar, E.J. (Eds.), Laser Radar Technology and Applications and Atmospheric Propagation XI, SPIE. p. 90800I. doi:10.1117/12.2050577.

Collin, A., Archambault, P., Long, B., 2008. Mapping the Shallow Water Seabed Habitat with the SHOALS. IEEE Transactions on Geoscience and Remote Sensing 46, 2947-2955. URL: http://ieeexplore. ieee. org/lpdocs/epic03/wrapper.htm?arnumber $=4595518$, doi:10.1109/tgrs. 2008.920020.

European Union, 2000. Directive 2000/60/ec of the European Parliament and of the Council of 23 October 2000 Establishing a Framework for Community Action the Field of Water Policy. Official Journal of the European Communities (OJL) L 327, 1-72.

Fuchs, E., Tuell, G., 2010. Conceptual Design of the CZMIL Data Acquisition System (DAS): Integrating a New Bathymetric Lidar with a Commercial Spectrometer and Metric Camera for Coastal Mapping Applications, in: Shen, S.S., Lewis, P.E. (Eds.), Algorithms and Technologies for Multispectral, Hyperspectral, and Ultraspectral Imagery XVI, SPIE. pp. 7695 - 7695 -12 . doi: $10.1117 / 12.851919$.

Girardeau-Montaut, D., 2018. Cloudcompare (version 2.10.alpha) [GPL Software] Retrieved Fom Http://www.cloudcompare.org/. URL: http://www. cloudcompare.org/. 
Guenther, G.C., 1985. Airborne Laser Hydrography System Design and Performance Factors. NOAA Professional Paper Series, National Ocean Service 1. URL: http://www.dtic.mil/cgi-bin/GetTRDoc?Location= U2\&doc $=$ GetTRDoc $\cdot$ pdf\&AD=ADA488936.

Guenther, G.C., Cunningham, A.G., LaRocque, P.E., Reid, D.J., 2001. Meeting the Accuracy Challenge in Airborne Lidar Bathymetry. Proceedings of EARSeL-SIG-Workshop LIDAR 1, 1-27.

Hartzell, P.J., Glennie, C.L., Finnegan, D.C., 2015. Empirical Waveform Decomposition and Radiometric Calibration of a Terrestrial Full-waveform Laser Scanner. IEEE Transactions on Geoscience and Remote Sensing 53 , 162-172. doi:10.1109/TGRS.2014.2320134.

Kinzel, P.J., Legleiter, C.J., Nelson, J.M., 2012. Mapping River Bathymetry With a Small Footprint Green LiDAR: Applications and Challenges1. JAWRA Journal of the American Water Resources Association 49, 183-204. doi:10.1111/jawr.12008.

Kinzel, P.J., Wright, C.W., Nelson, J.M., Burman, A.R., 2007. Evaluation of an Experimental LiDAR for Surveying a Shallow, Braided, Sand-bedded River. Journal of Hydraulic Engineering 133, 838-842. doi:10.1061/ (asce) 0733-9429 (2007)133:7 (838).

Kirmse, A., de Ferranti, J., 2017. Calculating the Prominence and Isolation of Every Mountain in the World. Progress in Physical Geography 41, 788-802. doi:10.1177/0309133317738163.

Klopfer, F., Hämmerle, M., Höfle, B., 2017. Assessing the Potential of a Lowcost 3-d Sensor in Shallow-water Bathymetry. IEEE Geoscience and Remote Sensing Letters 14, 1-5. URL: http://ieeexplore.ieee.org/ document/7970139/, doi:10.1109/LGRS.2017.2713991.

Legleiter, C.J., 2016. Inferring River Bathymetry Via Image-to-depth Quantile Transformation (IDQT). Water Resources Research 52, 3722-3741. doi:10. 1002/2016wr018730.

Legleiter, C.J., Overstreet, B.T., Glennie, C.L., Pan, Z., Fernandez-Diaz, J.C., Singhania, A., 2015. Evaluating the Capabilities of the CASI Hyperspectral Imaging System and Aquarius Bathymetric LiDAR for Measuring Channel Morphology in Two Distinct River Environments. Earth Surface Processes and Landforms 41, 344-363. doi:10.1002/esp. 3794

Legleiter, C.J., Roberts, D.A., Marcus, W.A., Fonstad, M.A., 2004. Passive Optical Remote Sensing of River Channel Morphology and In-stream Habitat: Physical Basis and Feasibility. Remote Sensing of Environment 93, 493-510. doi:10.1016/j.rse. 2004.07.019.

Maas, H.G., 2015. On the Accuracy Potential in Underwater / Multimedia Photogrammetry. Sensors 15, 18140-18152. URL: http://www.mdpi . com/1424-8220/15/8/18140, doi:10.3390/s150818140.

Maddock, I., 1999. The Importance of Physical Habitat Assessment for Evaluating River Health. Freshwater Biology 41, 373-391. doi:10.1046/j. 1365-2427.1999.00437.x.

Mandlburger, G., 2017. Interaction of Laser Pulses with the Water Surface Theoretical Aspects and Experimental Results. Allgemeine VermessungsNachrichten (AVN) begutachtete Beiträge 11-12, 343-352.

Mandlburger, G., Hauer, C., Wieser, M., Pfeifer, N., 2015. Topo - Bathymetric Lidar for Monitoring River Morphodynamics and Instream Habitats - a Case Study at the Pielach River. Remote Sensing 7, 6160 6195. URL: http://www.mdpi.com/2072-4292/7/5/6160, doi:10. 3390/rs70506160.

Mandlburger, G., Otepka, J., Karel, W., Wagner, W., Pfeifer, 2009. Orientation and Processing of Airborne Laser Scanningdata (OPALS) - Concept and First Results of a Comprehensive Als Soft-ware, in: International Archives of the Photogrammetry, RemoteSensing and Spatial Information Sciences38 (Part 3/W8), pp. 55-60.

Mandlburger, G., Pfeifer, N., Soergel, U., 2017. Water Surface Reconstruction in Airborne Laser Bathymetry from Redundant Bed Observations. ISPRS Annals of Photogrammetry, Remote Sensing and Spatial Information Sciences IV-2/W4, 123-130. doi:10.5194/ isprs-annals-IV-2-W4-123-2017.

Mandlburger, G., Pfennigbauer, M., Pfeifer, N., 2013. Analyzing near Water Surface Penetration in Laser Bathymetry - a Case Study at the River Pielach. ISPRS Annals of Photogrammetry, Remote Sensing and Spatial Information Sciences II-5/W2, 175-180. doi:10.5194/ isprsannals-ii-5-w2-175-2013.
Mandlburger, G., Pfennigbauer, M., Steinbacher, F., Pfeifer, N., 2011. Airborne Hydrographic LiDAR Mapping - Potential of a New Technique for Capturing Shallow Water Bodies, in: 19th International Congress on Modelling and Simulation, pp. 55-60. URL: http://mssanz.org.au/modsim2011/ E14/mandlburger.pdf.

Mitchell, S.E., Thayer, J.P., 2014. Ranging through Shallow Semitransparent Media with Polarization Lidar. Journal of Atmospheric and Oceanic Technology 31, 681-697. doi:10.1175/jtech-d-13-00014.1.

Pe'eri, S., Philpot, W., 2007. Increasing the Existence of Very Shallow - Water LIDAR Measurements Using the Red-channel Waveforms. IEEE Transactions on Geoscience and Remote Sensing 45, 1217-1223. doi:10.1109/ tgrs.2007.894584.

Pfennigbauer, M., Rieger, P., Studnicka, N., Ullrich, A., 2009. Detection of Concealed Objects with a Mobile Laser Scanning System, in: Turner, M.D., Kamerman, G.W. (Eds.), Laser Radar Technology and Applications XIV, SPIE. pp. 7323 - 7323 - 9. doi:10.1117/12.828293.

Pfennigbauer, M., Ullrich, A., 2010. Improving Quality of Laser Scanning Data Acquisition through Calibrated Amplitude and Pulse Deviation Measurement, in: Turner, M.D., Kamerman, G.W. (Eds.), Laser Radar Technology and Applications XV, SPIE. pp. 76841F-76841F-10. doi:10.1117/ 12.849641

Pfennigbauer, M., Wolf, C., Ullrich, A., 2013. Enhancing Online Waveform Processing by Adding New Point Attributes, in: Turner, M.D., Kamerman, G.W. (Eds.), Laser Radar Technology and Applications XVIII, SPIE. pp. 873104-873104-9. doi:10.1117/12.2015733.

RIEGL, 2018. RiPROCESS, Datasheet from http://www.riegl.com/. URL: http://www.riegl.com/uploads/tx_pxpriegldownloads/11_ Datasheet_RiProcess_2016-09-16_01.pdf.

Sahalan, M., Idris, M.M., Abidin, Z., Kamarudin, M.C., 2016. Tilt Compensated Mechanical Measurement Mechanism for Very Shallow Water USV Bathymetry, in: 2016 IEEE International Conference on Underwater System Technology: Theory and Applications (USYS), IEEE. pp. 48-54. URL: http://ieeexplore.ieee.org/document/7893933/, doi:10.1109/USYS. 2016.7893933

Schwarz, R., Pfeifer, N., Pfennigbauer, M., Ullrich, A., 2017. Exponential Decomposition with Implicit Deconvolution of Lidar Backscatter from the Water Column. PFG - Journal of Photogrammetry, Remote Sensing and Geoinformation Science 85, 159-167. URL: http://link. springer . com/10. 1007/s41064-017-0018-z, doi:10.1007/s41064-017-0018-z.

Schwarz, R., Pfennigbauer, M., 2018. Finding Model Parameters for the System Waveform of a Full-wave Lidar: A Pragmatic Solution, in: Bostater, C.R., Mertikas, S.P., Neyt, X. (Eds.), Remote Sensing of the Ocean, Sea Ice, Coastal Waters, and Large Water Regions 2018, SPIE. pp. 107840A-1107840A-8. doi:10.1117/12.2323965.

Shen, K., Lu, H., Baig, S.A., Jiang, G., McManus, J.W., Wang, M.R., 2017. Laser-based Water Depth Measurement System Deployed Via Unmanned Aerial Vehicle. Journal of Applied Remote Sensing 11, 032409. doi:10. 1117/1.jrs.11.032409

Smith, M.W., Cox, N.J., Bracken, L.J., 2011. Modeling Depth Distributions of Overland Flows. Geomorphology 125, 402-413. doi:10.1016/ j.geomorph.2010.10.017.

Steinbacher, F., Aufleger, M., 2013. Airborne Hydromapping: A New Approach to Manage Hydraulics, Morphology and Ecology. International journal on hydropower and dams 20, 74-77.

Wagner, W., Ullrich, A., Ducic, V., Melzer, T., Studnicka, N., 2006. Gaussian Decomposition and Calibration of a Novel Small-footprint Full-waveform Digitising Airborne Laser Scanner. ISPRS Journal of Photogrammetry and Remote Sensing 60, 100-112. doi:10.1016/j . isprs jprs . 2005.12. 001

Westaway, R., Lane, S., Hicks, D., 2001. Remote Sensing of Clear-water, Shallow, Gravel-bed Rivers Using Digital Photogrammetry. Photogrammetric Engineering and Remote Sensing 67, 1271-1281.

Zhao, X., Zhao, J., Zhang, H., Zhou, F., 2018. Remote Sensing of Suspended Sediment Concentrations Based on the Waveform Decomposition of Airborne LiDAR Bathymetry. Remote Sensing 10, 247. doi:10.3390/ rs10020247. 